\title{
Erratum: Phase synchronization and intermittent behavior in healthy and Alzheimer-affected human-brain-based neural network [Phys. Rev. E 99, 022402 (2019)]
}

\author{
R. C. Budzinski, B. R. R. Boaretto, T. L. Prado, and S. R. Lopes
}

(C) (Received 11 March 2019; published 4 June 2019)

DOI: 10.1103/PhysRevE.99.069901

Due to a misinterpretation of the rescaled matrix generated in Ref. [1], we suggested that the rescaled matrices should be representative of each group of patients (healthy and Alzheimer affected) since we thought each matrix is a mean value over a group of patients. However, the correct interpretation is that each matrix is just a single example, so despite the correctness of our methodology, the text leads the readers to think the results can be interpreted as general, but they are not. This fact does not invalidate our results, but it weakens our conclusions. In the interest of clarity, we call attention to this point.

[1] F. A. S. Ferrari, R. L. Viana, A. S. Reis, K. C. Iarosz, I. L. Caldas, and A. M. Batista, Physica A 496, 162 (2018). 